\title{
Evaluation of LPWAN Technologies for Smart Cities: River Monitoring Use-case
}

\author{
Wael Guibene, Johannes Nowack, Nikolaos Chalikias ${ }^{+}$, Kevin Fitzgibbon ${ }^{+}$, Mark Kelly, David Prendergast \\ Intel Labs, Leixlip-Ireland ; ${ }^{+}$Nimbus Centre/Cork Institute of Technology, Cork-Ireland \\ contact author: wael.guibene@intel.com
}

\begin{abstract}
This paper presents the results of the joint Intel/ Nimbus Low Power-Wide Area (LPWA) technology PoC deployment in smart cities context. The PoC deployment addresses the problem of river Liffey monitoring in Dublin city center. We deployed a buoy on the Liffey river in Dublin for duration of 8 months. The deployed buoy embeds many sensors inside the hull enclosure and outside. The data captured is, from the water: depth, temperature and velocity; from inside the hull: temperature, humidity and barometric pressure; from the GPS unit: location and timestamp; and from the system: battery voltage. The buoy also embeds a LoRa-based LPWA transceiver and a $3 G$ modem for backup. The paper gives an insight of the results obtained in terms of range and data consistency and gives conclusions on the use of LPWA technologies in the context of smart cities.
\end{abstract}

\section{INTRODUCTION AND CONTEXT}

In 2014 Intel and Dublin City Council (DCC) agreed to collaborate to create an Internet of Things Demonstrator for Smart City Services and Solutions around Public Safety[1]. The objective of the project is to provide a solution for collecting and analyzing environmental and potentially other data within Dublin through sensor technology and combining this data with relevant environmental policy input and active participation from council employees, citizens and key stakeholders around public safety.

Through a series of stages over the course of four years, the deployment involves the installation of a range of sensors and gateways across Dublin with an integrated Intel End-to-End (from Gateway to Cloud) software platform which will allow secure gateway management, data capture, data transfer and data analytics and presentation. Key objectives will include:

- Deploy Low Powered Wide Area (LPWA) and Intel Quark based IoT gateways across Dublin with an integrated Intel End-to-End (from gateway to Cloud) software platform which will allow secure gateway management, data capture, data transfer, analytics and presentation

- Develop use cases and opportunities through the platform for cost reduction and service, security and quality of life improvement for Dublin citizens and stakeholders

- Explore operational, business, service and citizen engagement models for next generation Internet of Things solutions and support innovation in the city

This paper shares results from an eight month trial of a low cost LPWA and Intel Edison enabled river monitoring buoy [2] built by a joint Nimbus /Cork Institute of Technology and Intel team to compare LoRa vs $3 \mathrm{G}$ transmissions of data on river depth, air and water temperature, barometric pressure and solar/battery performance. The site for the deployment was selected by DCC to provide information on river conditions on a section of the River Liffey that had previously overtopped, flooding a nearby underground car park.

The preliminary phase of the project involved working with city council officials and departments to scope out use cases addressing major challenges of significant relevance to Dublin City Council and aligned to its Smart Dublin Initiative. Broadly this agenda includes transportation, environment and safety and energy efficiency; but the decision was taken to initially focus on exploring the potential of emergent LPWA technologies and network capabilities to enhance and scale existing flood monitoring solutions.

The rest of the paper is organized as follows: in section II, we provide an overview of the Nimbus/Intel buoy. In section III, we present the system end-to-end architecture and the frame structure used for communication between the buoy and the cloud back-end system. In section IV, we discuss the obtained results over the eight month experiment and we identify the erroneous frames contaminating the dataset. Section V concludes with the results obtained through the experiment and presents future directions to make the deployment more reliable and secure.

\section{INTEL/NIMBUS BUOY OVERVIEW}

The sensor platform is a fully enclosed and sealed custommade boat-shaped hull or "buoy", designed for fast-flowing river deployments.

Power is provided by $3 \mathrm{nr}$ solar PV panels mounted on the roof of the buoy, which charge $2 \mathrm{nr} 12 \mathrm{~V}$ sealed lead-acid batteries. Nimbus designed and built a number of modular electronic circuit boards for communications, sensing, maximum powerpoint tracking from the PV panels, power management, and to host embedded CPUs, compatible with the Nimbus "n-Blocks" system.

The electronics have been scheduled for "sleep" mode most of the time to save power. The low-power scheduling CPU is always powered on, and wakes up the rest of the system periodically, to capture data from the various sensors and communicate via LoRa every 10 minutes; and to send 3G signals every 12 hours, since the communication method is power-hungry and costly. The $3 \mathrm{G}$ mode transmits via a direct internet connection to the "Thingspeak" open-source cloudbased sensor/data platform while the LoRa transceiver 


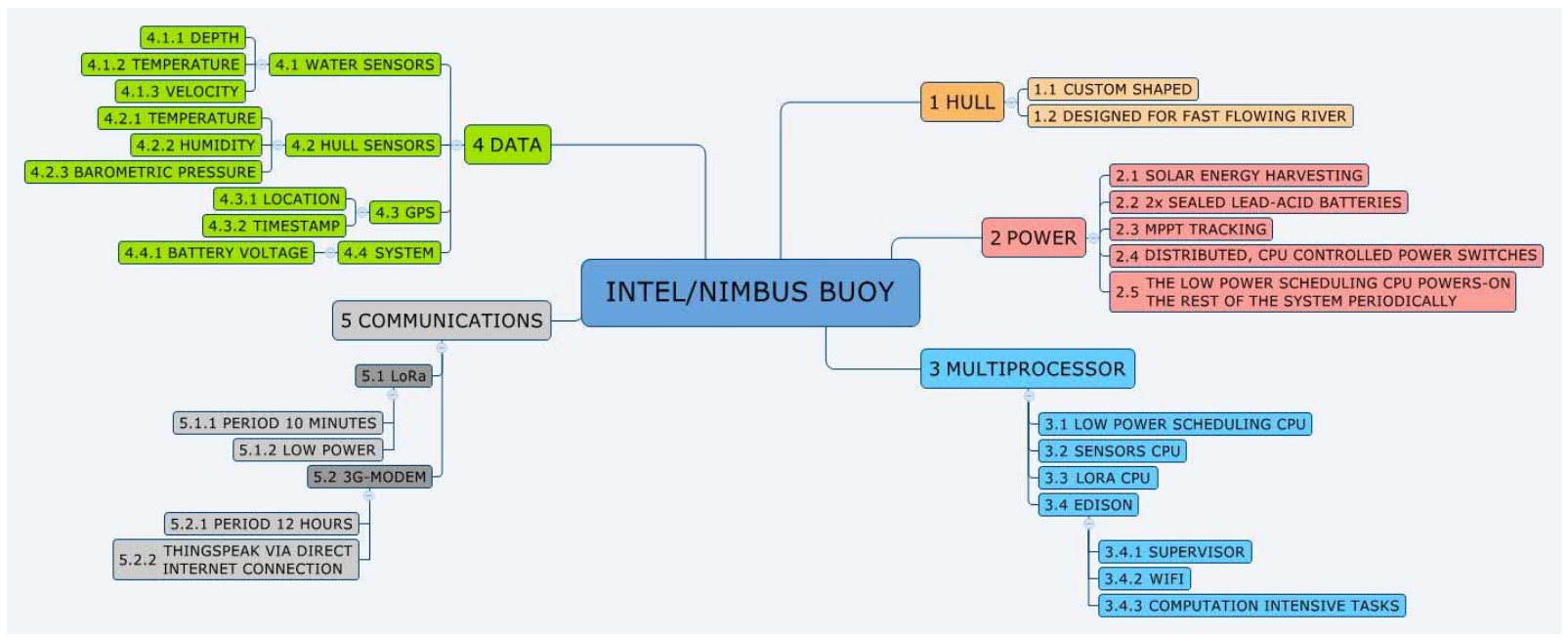

(a) Intel/Nimbus Buoy Architecture
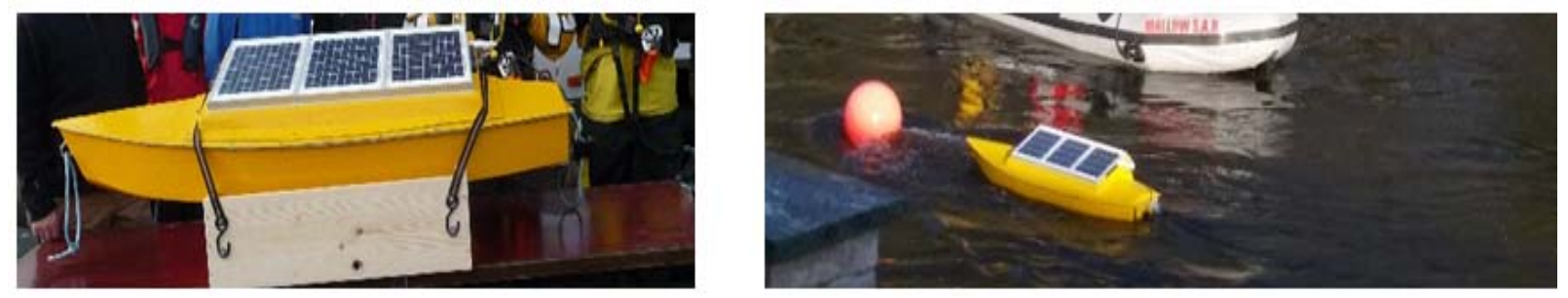

(b) Intel/Nimbus Buoy Deployment

Fig. 1: Intel/Nimbus Buoy Architecture and Deployment Overviews

pushes data to the Intel backend system described in Section III. The Sensor CPU communicates with all the sensors, and controls the communications. The LoRa CPU implements lowpower proprietary protocol LoRa communications. An Intel Edison unit supervises the system, provides Wi-Fi connectivity and carries out the computationally intensive tasks.

The data captured is: from the water: depth, temperature and velocity; from inside the hull: temperature, humidity and barometric pressure; from the GPS unit: location and timestamp; and from the system: battery voltage.

\section{END-TO-END SYSTEM ARCHITECTURE}

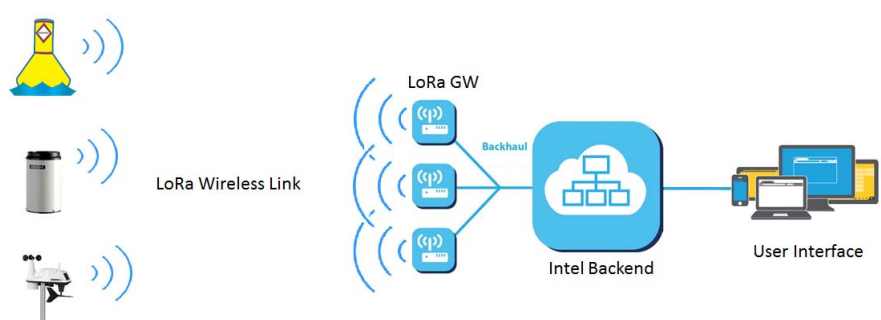

Fig. 2: Deployment E2E Architecture based on LoRa Technology
During the deployment, some rainfall gauges and waterlevel (ultrasonic) sensors as well as the Intel/Nimbus Buoy were deployed across Dublin city center. The overall end-toend system architecture is depicted in Figure (2).

The deployed buoy and sensors report frequently, within their duty-cycle limitations, their measurements. The transmitted frames are received by the gateways deployed in Dublin city center as well as on the Three Rock gateway.

Throughout our experiment, we used LoRa [3], [4] (Long Range) technology from Semtech. LoRa is a physical layer specification based on chirp spread spectrum (CSS) with integrated Forward Error Correction (FEC). Transmissions use a wide band $(125 \mathrm{KHz})$ to be more resilient to interference and to handle frequency offsets due to low cost crystals. A LoRa receiver can decode transmissions $19.5 \mathrm{~dB}$ below the noise floor. Thus achieving very long communication distances. LoRa key properties are: long range, high robustness, multipath resistance, Doppler resistance and low power. LoRa operates in the lower ISM bands (EU: $868 \mathrm{MHz}$ and 433 MHz, USA: $915 \mathrm{MHz}$ and $433 \mathrm{MHz}$ ). A LoRa radio has four configuration parameters: carrier frequency, spreading factor, bandwidth and coding rate. The selection of these parameters determines energy consumption, transmission range and resilience to noise. The used configuration is as follows: 


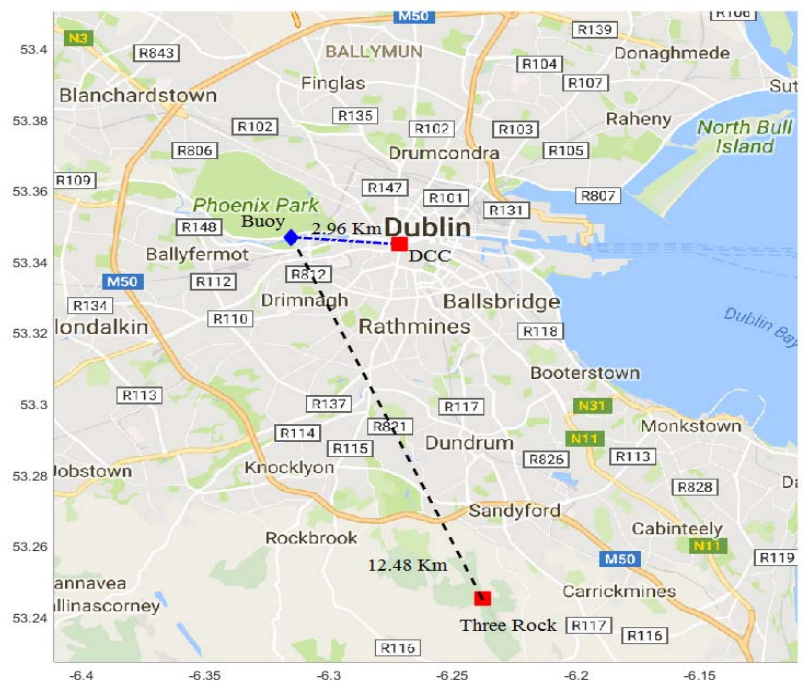

(a) PoC Deployment Map

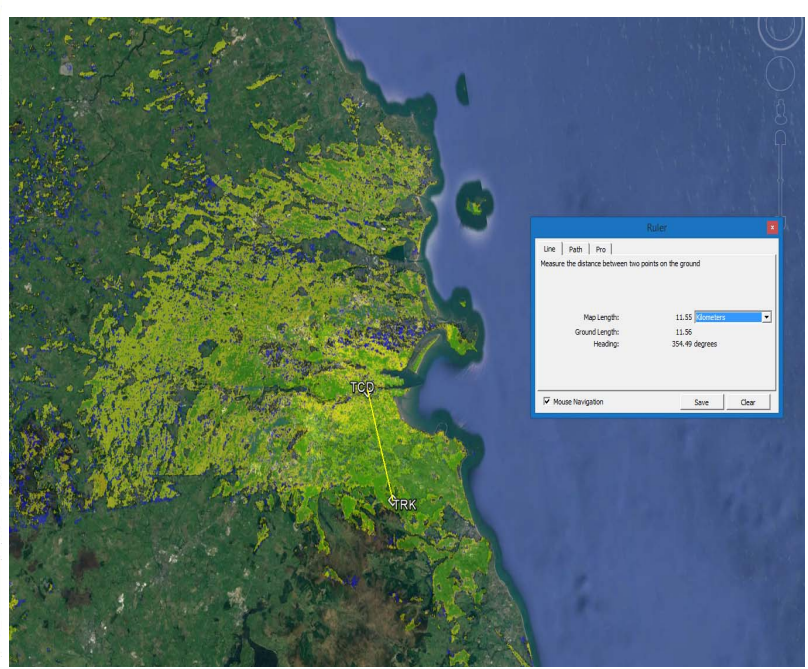

(b) LoRa Theoretical Coverage Using 2 GWs

Fig. 3: PoC and Theoretical Deployment Maps

\begin{tabular}{|c|c|}
\hline Parameter & Used Value \\
\hline Frequency & $869.525 \mathrm{MHz}$ \\
Spreading Factor & SF12 \\
Transmit Power & $17 \mathrm{dBm}$ \\
Bandwidth & $125 \mathrm{KHz}$ \\
Codig Rate & $4 / 5$ \\
\hline
\end{tabular}

The gateways are forwarding the received frames to an AWS-hosted cloud network server (CNS).

The CNS proceeds to a first filtering of the received frames over the over-the-air. At this stage, only frames with a valid CRC are processed. From the AWS-based CNS we push data through MQTT to a processing pipeline. The latter consists of an OpenTSDB that consumes sensor values and stores them in a database. We also implemented a UI and a series of API to pull and display data from the OpenTSDB.

When storing data at the OpenTSDB,we apply a second level of filtering obtained by applying boundary conditions to sensor readings and values. The need to apply these boundary conditions came from the fact that we were seeing errors on sensor reading, coming either from intermittent fault on the sensor itself or over-the-air bytes changing but coming with a good CRC that result in erroneous sensor data.

As the focus of the paper is the buoy deployment, in the rest of the paper results are only shown for buoy data. Figure (3-a) depicts the location where the buoy and the gateways were positioned for the first PoC. We used two gateways to validate both urban and near line of sight (near-LOS) long range environment.

Figure (3-b) depicts the theoretical coverage for the considered scenario. The two gateways installed in Three Rock and on top of Dublin City Council building can cover a huge area in the Dublin City and its suburbs.

The buoy is at an altitude of $4-5 \mathrm{~m}$ above sea level and is located $3 \mathrm{Km}$ to the first gateway (DCC). The DCC gateway is at an altitude of $55 \mathrm{~m}$ above sea level. Both the GW and the buoy are in an urban highly populated areas, characterized by thick walls and old Irish buildings. The second GW is is located at Three Rock, in a long range near-LOS environment $13 \mathrm{Km}$.

The main transmission site on Three Rock is owned and operated by $2 \mathrm{RN}$ with a $140 \mathrm{~m}$ mast close to the peak, which is 448 meters above sea level, which means the GW at Three Rock is almost at $580 \mathrm{~m}$ above sea level. Even though Three Rock gateway is on a high point, it is not directly overlooking the buoy and the path is also an urban long range scenario.

A fixed-size packet of 64 bytes is sent by the buoy LoRa transmitter. An example of the 64-byte datagram is as follows, with all values in hexadecimal:

$\begin{array}{llllllllllllllll}00 & 00 & 00 & 00 & 32 & 81 & 5 \mathrm{C} & 56 & 24 & 00 & 00 & 00 & 33 & 33 & 33 & 3 \mathrm{~F}\end{array}$ 00000000 CD CC A0 4100000242 9C 09 A1 45 CB 09 $50 \mathrm{C} 400$ 0C C7 473333 B5 41 AC 1C 2442 AE 474551 000000000000000000000000

The structure of the packet is shown in the tables below. All parameters are in $l s b$ first (aka little-endian form). 


\begin{tabular}{|c|c|c|}
\hline \multicolumn{3}{|c|}{ Buoy ID (Bytes 0-3) } \\
\hline Parameter & Example Value & Bytes (Hex) \\
\hline 0 & 0 & 00000000 \\
\hline \multicolumn{3}{|c|}{ 32-bit Unsigned Integers (Bytes 4-11) } \\
\hline Parameter & Example Value & Bytes (Hex) \\
\hline Timestamp (in seconds) & 1448902962 & $32815 \mathrm{C} 56$ \\
\hline Reset Count & 36 & 24000000 \\
\hline \multicolumn{3}{|c|}{ 32-bit Floats (Bytes 12-63) } \\
\hline Parameter & Example Value & Bytes (Hex) \\
\hline Depth (m) & 0.7 & $3333333 \mathrm{~F}$ \\
\hline Flow Rate (m/s) & 0 & 00000000 \\
\hline Water Temperature (C) & 20.1 & CD CC A0 41 \\
\hline Altitude & 32.5 & 00000242 \\
\hline Latitude & 5153.201 & 9C 09 A1 45 \\
\hline Longitude & -832.153 & CB $0950 \mathrm{C} 4$ \\
\hline Pressure & 101912 & 00 0C C7 47 \\
\hline Buoy Temperature $(\mathrm{C})$ & 22.65 & 3333 B5 41 \\
\hline Buoy Humidity & 41.028 & AC 1C 2442 \\
\hline Battery Level (V) & 12.33 & AE 474541 \\
\hline Spare Data Field 1 & 0 & 00000000 \\
\hline Spare Data Field 2 & 0 & 00000000 \\
\hline Spare Data Field 3 & 0 & 00000000 \\
\hline
\end{tabular}

\section{EXPERIMENTAL RESULTS}

In Figures $(4,5,6,7,8)$, we present the results for the raw, real-time acquisitions over 8 months of respectively the voltage reading, the temperature in the buoy, the water temperature, the atmospheric pressure and the water depth (blue curves). The figures are complemented with a moving average filter to smoothen the curves and help understand the trend of the curves (red curves).

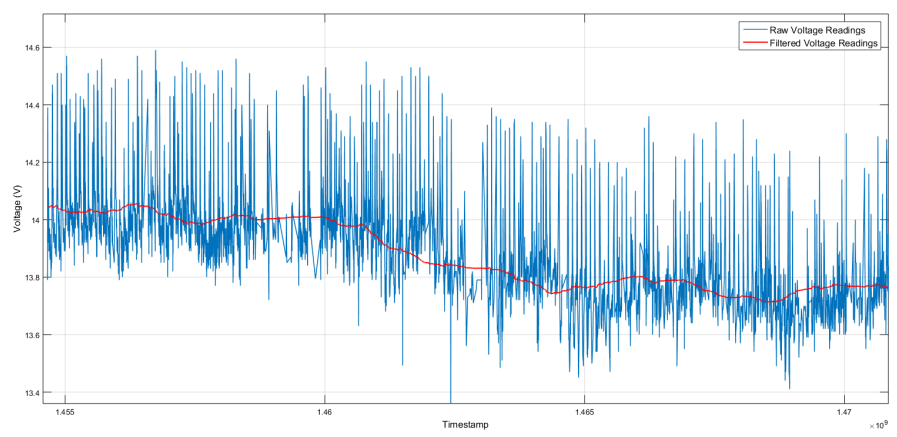

Fig. 4: Evolution of Battery Voltage

The results are coherent with expectations, as the voltage curve, Fig (4), is dropping over the 8 month experiment running from January 2016 to August 2016. Both internal and water temperature curves, respectively Fig (5) and Fig (6) are also consistent with the expected behavior, as from January to August the temperature is increasing. The reading on the internal temperature is high frequency (presenting more spikes) as we suspect that the electronic components heating contributes to making the temperature fluctuate in the buoy.

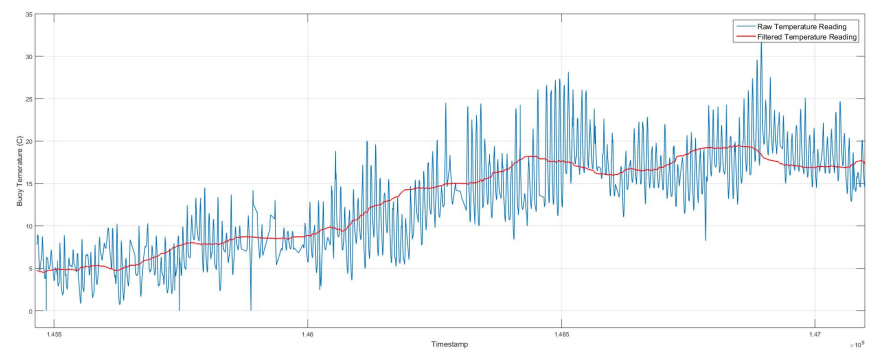

Fig. 5: Evolution of Buoy Temperature

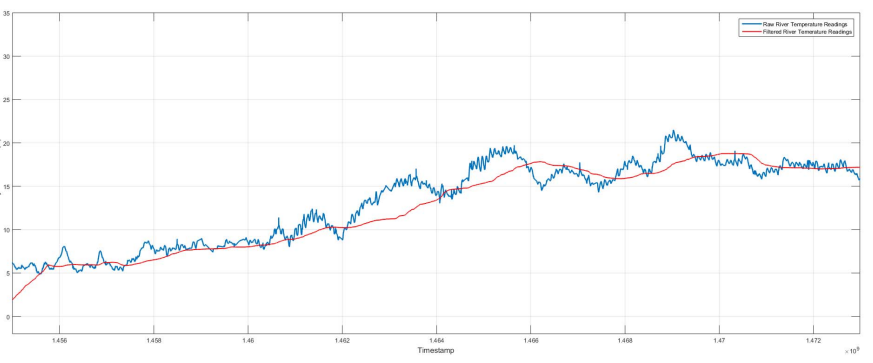

Fig. 6: Evolution of Water Temperature

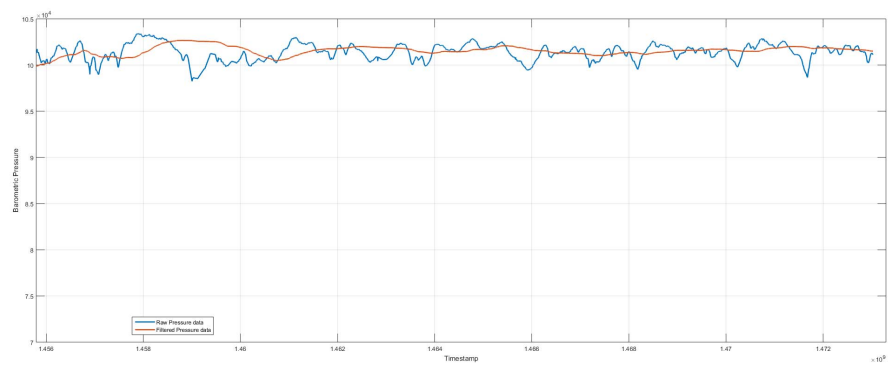

Fig. 7: Evolution of Measured Atmospheric Pressure

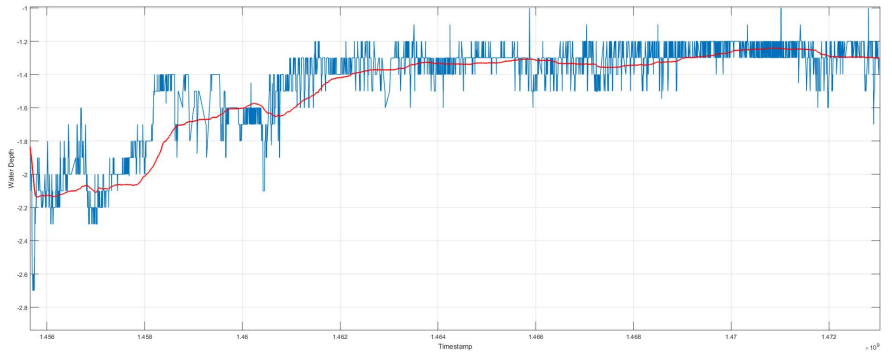

Fig. 8: Evolution of Measured River Depth 
The pressure in Fig (7) fluctuates in an around the $105 \mathrm{~Pa}$, which is the expected value. The reported Figures still contain erroneous values as only the first filtering based on CRC is applied at this stage. An easy way to count the errors in the second filtering is by identifying packets where spare Data Field 1, Data Field 2 and Data Field 3 are non zeros. This count gives an over all error of 102 data-point over a total 5745 data-point or a ratio of $1.78 \%$ for the 8 month experiment.

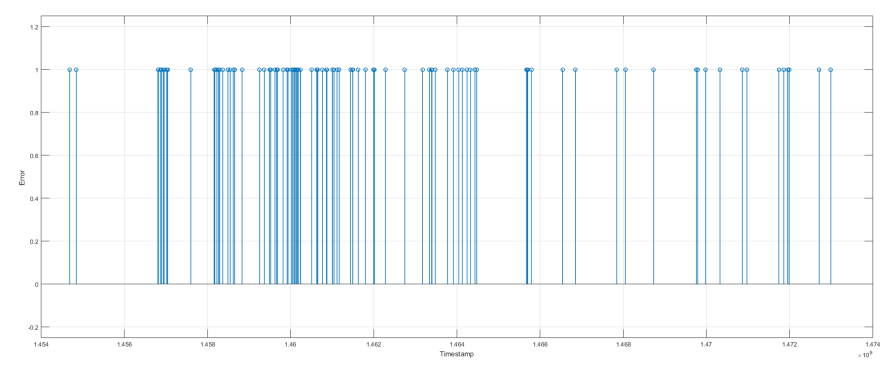

Fig. 9: Distribution of Error Measurements Through the Experiment: $1.78 \%$

\section{CONCLUSIONS}

In this paper, we presented the results for our first 8 month deployment of smart city scenario based on low powerwide area technologies (LoRa). The results obtained from the deployment are overall satisfactory and are coherent with the expected trends. The deployment could have been more satisfactory if we used a more robust MAC Layer (LoRaWAN [5]) approach rather than relying just on the PHY Layer. In the next phase, we envisage to make our approach more robust by adopting a MAC layer (LoRaWAN, Symphony Link, 802.15.4e) on top of the LoRa modulation to minimize the effects of wireless channel on the transmitted data. We also will adopt shorter messages by either fragmenting the 64 Bytes payload or adopting an adaptive data-rate approach that minimizes time on air while guaranteeing the best coverage function of the endpoint RSSI and SINR. The security aspect has also to be considered for the next iteration and will be addressed by either LoRaWAN E2E AES128 encryption or adding an extra layer of encryption on the payload itself.

\section{ACKNOWLEDGMENT}

The authors would like to thank and acknowledge the help provided by Dublin City Council (DCC) while running the experiment and also 2RN Ireland for gateways deployments.

\section{REFERENCES}

[1] Silicon Republic, online edition - Apr, 2014: Intel to turn Dublin into worlds first Internet of Things city.

[2] The Independent, online edition - Mar, 2014: Oh buoy! Whats that?

[3] Seller, O.B. and Sornin, N., Semtech Corporation, 2016. Low power long range transmitter. U.S. Patent 9,252,834.

[4] Guibene, W; Sornin, N; Enabling M2M Communications and IoT Applications through LoRa Technology, In 2014 IEEE Globecom Industry Forum (Semtech Corporation Demo)

[5] Sornin, N., Luis, M., Eirich, T., Kramp, T. and Hersent, O., 2015. LoRaWAN Specification v1.0. LoRaAlliance Delivrable 